\title{
Addressing and Managing the Increasing Rate of Obesity among Canadian Youth
}

\author{
Sofia Huroy (University of Toronto) \\ News Reporter (HSI 2011-2012)
}

The obesity epidemic continues to spread at an alarming rate. Since the late 1970s, there has been a significant rise in the number of overweight and obese people. This rise is seen in many parts of the world, across all age groups and in both genders. And Canada is no exception. Recent Statistics Canada data have shown that 26\% of Canadian youth aged 2-17 years are overweight or obese. With a growing population of overweight or obese children, there is strong pressure to implement prevention programs that promote healthy nutrition and increased physical activity among children. Therefore, preventing childhood obesity has recently become a priority among different governments, including Canada. Surprisingly, until about 25 years ago, type 2 diabetes was uncommon in children and adolescents, and rarely seen in individuals under the age of 40 . As obesity becomes more prevalent in younger populations, without intervention, these youth face serious health complications in the future. This will no doubt cause further strain on Canada's healthcare system.

\section{STOMP provides various types of treatments such as cognitive behavioural therapy, medi- cations, specialized diet and bariatric surgery.}

Obesity is a complex condition. Multiple factors such as genetics, lifestyle, diet, overall physical and emotional health, and race/ethnicity can influence the development of obesity in children and adolescents. Other factors such as television viewing, and advertising have also been found to play a role in obesity development. Obesity is associated with increased risk to cardiovascular disease, hypertension, type II diabetes mellitus, menstrual irregularities, obstructive sleep apnea, to name a few.

Experts agree the best method to prevent obesity is to start early. Across Canada multiple paediatric Weight

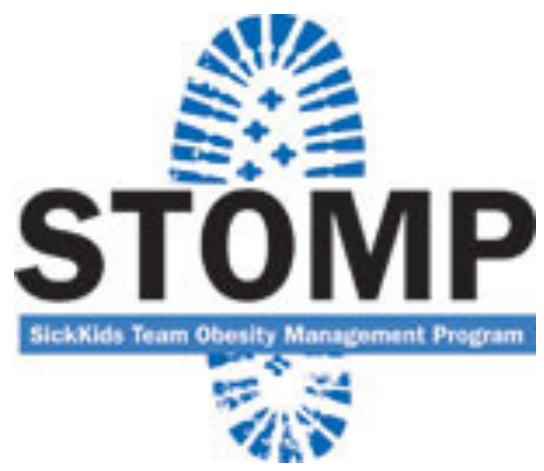

Across Canada multiple paediatric Weight Management Programs have been put in place to combat and treat obesity in youth. One such program is the STOMP (SickKids Team Obesity Management Program), established at The Hospital for Sick Children (SickKids) in 2010.

Management Programs have been put in place to combat and treat obesity in youth. One such program is the STOMP (SickKids Team Obesity Management Program), established at The Hospital for Sick Children (SickKids) in 2010.

Dr. Catherine Birken is a general paediatrician at SickKids, and co-director of STOMP. She reveals the idea of STOMP came into existence about 5 years ago. Overweight/obese children were being assessed by many specialists all over the hospital, with each specialist addressing one particular issue. That is because some children also had significant obesity-related co-morbidities or significant co-existing chronic illnesses such as tumours or medications induced obesity. Thus STOMP came about, to address all health issues of the child/adolescent as a team. When the idea was brought forward to the Ontario Ministry of Health and Long-Term Care, the ministry agreed to fund it for 3 years, with enough funding for 50 children per year.

All children enrolled in STOMP must be referred by a Health Care Professional, and have a BMI > 99th percentile, or a $\mathrm{BMI}>$ 95th percentile plus a significant obesity-related co-morbidity. Then, the adolescents will be assessed by the STOMP team before joining the program. The STOMP healthcare professional team includes a general paediatrician, a paediatric endocrinologist, a surgeon, a nurse practitioner, a dietician, an exercise therapist, a psychologist, a social worker, and collaborations with 
other healthcare professionals with various specialties (e.g. adolescent medicine and cardiology).

STOMP is a two-year intensive program. Along with the healthcare professionals, the adolescent group between the ages of 12-17 years partake in weekly group sessions. A major focus of the program is psychological and mental health support in a group setting. The caregivers of the patients also have their own group sessions. Dr. Birken shares that "[STOMP] is fortunate to have significant mental health, dietetics and physical activity expertise, and if affective there is an opportunity to expand."

Countless studies have shown that obesity affects mental health as well as physical health. Adolescent obesity, like adult obesity, is associated with numerous psychological problems. Obese children and adolescent are also teased, and as a result suffer from low self-esteem, nega-tive body image, and depression. Furthermore, obese individuals have been shown to be discriminated against employment and educational opportunities. ${ }^{1}$ Stigma against weight gain is further exuberated through the media. The media often portrays being thin with positive messages, while being overweight with negative messages. Thus, Dr. Birken explains STOMP is unique because it is "a program designed specifically for adolescents... there are not many programs out there for adolescents who suffer from morbid obesity." She further adds that due to the severity of health among the children, the program is "very psychology based...heavy on mental health."

STOMP provides various types of treatments such as cognitive behavioural therapy, medications, specialized diet and bariatric surgery. However, Dr. Birken clarifies that "only a small part of the program is surgery." Less than 10 children have had surgery so far. She points out that "[STOMP has] a rigorous process to identify children who are good candidates for surgery." To qualify patients must show willingness to lifestyle changes after surgery. Some of the criteria to have surgery include that the adolescents must have finished growing, i.e. around $16-18$ years of age, and consent and support of family to be successful.
The social and psychological impact of obesity, especially on females, is staggering. Adolescent girls are constantly bombarded with messages that a slender body is more attractive and desirable. As such, body dissatisfaction is more common in females. This can lead to eating disorders and other mental health problems. ${ }^{2,3}$ Dr. Birken agrees that there is a "huge bias" towards overweight/ obese individuals, but points out that the outcome of the surgeries is "not always affective" and it is not a "tool to be used to just become thin." She shares that "in STOMP we are concerned with health outcomes, not only BMI. These outcomes include improved quality of life, school attendance, as well as reduced obstructive sleep apnea, and high cholesterol."

For the future, Dr. Birken would like to "evaluate the outcomes [of STOMP] ... [and asses the] mental health and school outcomes" of the children involved with STOMP. If the program is considered successful, she would like "to advocate for more resources, and expand the program." One area she would like to expand the program is to children with mental disability but who also suffer from obesity. She states, with the current model of STOMP such individuals "can't participate in group therapy, but [stresses] that is not the only form of therapy."

Youth are an important population for obesity prevention. In addition, the probability that childhood obesity will persist into adulthood increases in adolescence. Hence, Weight Management Programs like STOMP play a key role in attempting to combat obesity early on.

\section{References}

1. Puhl, R.M. and J.D. Latner, Stigma, obesity, and the health of the nation's children. Psychological bulletin, 2007. 133(4): p. 557-80.

2. Neumark-Sztainer, D., et al., Does body satisfaction matter? Five-year longitudinal associations between body satisfaction and health behaviors in adolescent females and males. The Journal of adolescent health : official publication of the Society for Adolescent Medicine, 2006. 39(2): p. 244-51.

3. Paxton, S.J., et al., Body dissatisfaction prospectively predicts depressive mood and low self-esteem in adolescent girls and boys. Journal of clinical child and adolescent psychology : the official journal for the Society of Clinical Child and Adolescent Psychology, American Psychological Association, Division 53, 2006. 35(4): p. 539-49.

\section{Sofia Huroy}

Sofia Huroy is currently pursuing a MSc degree in Pharmaceutical Sciences at the University of Toronto, where she also received her Honours BSc. Her research is in the area of neuroscience, and focuses on the use of animal models to study the mechanism of opiate dependent tolerance. 